\title{
Advances in the Application of Fondaparinux in Acute Coronary Syndrome
}

\author{
Xue Han ${ }^{1}$, Lijun Jin ${ }^{2 *}$ \\ ${ }^{1}$ Yangtze University, Jingzhou, China \\ ${ }^{2}$ The First Affiliated Hospital of Yangtze University, Jingzhou, China \\ Email:634009124@qq.com, ^2690465736@qq.com
}

How to cite this paper: Han, X. and Jin, L.J. (2020) Advances in the Application of Fondaparinux in Acute Coronary Syndrome. Case Reports in Clinical Medicine, 9, 201-207. https://doi.org/10.4236/crcm.2020.97028

Received: June 22, 2020

Accepted: July 14, 2020

Published: July 17, 2020

Copyright (c) 2020 by author(s) and Scientific Research Publishing Inc. This work is licensed under the Creative Commons Attribution International License (CC BY 4.0).

http://creativecommons.org/licenses/by/4.0/

\begin{abstract}
Acute Coronary Syndrome (ACS) is one of the major causes of death worldwide, including unstable angina, ST-segment elevation myocardial infarction and NST-segment elevation myocardial infarction. ACS refers to a series of life-threatening heart diseases, which is caused by rupturing coronary plaque and releasing thrombin activation. Then thrombin is activated and generates plaque and thrombosis, which increases the risk of cardiac death and myocardial infarction. Aggressive and conservative treatment is available in clinic practice. Anticoagulant therapy is usually the first choice for conservative treatment and used in combination with dual antiplatelet drugs, which plays an important role in the treatment of acute coronary syndrome. Fondaparinux as a commonly used anticoagulant drug is both antithrombotic effectively and can reduce the risk of bleeding and coronary microvascular dysfunction in the pathogenesis of ischemic heart disease. However, it increased the rate of bleeding. People pay more attention to the role of long-term prognosis. Domestic and foreign researches contrast outcomes of acute coronary syndrome of fondaparinux and low molecular weight heparin.
\end{abstract}

\section{Keywords}

Fondaparinux Sodium, Acute Coronary Syndrome, Heparin

\section{Introduction}

Acute coronary syndrome is still a serious challenge to the clinical practice [1] [2] [3] [4]. Each person experiences a coronary event every 40 seconds, which increases the number of recurrent myocardial infarction by more than 600,000 and 200,000 per year [5] [6]. Although great progress has been made in the treatment of ACS in recent years, the survival rate has been improved. The mor- 
tality rate of cardiovascular diseases is still relatively high [4].

As an anti-Xa factor drug, fondaparinux sodium has complete bioavailability [5]. It can be administered once a day through renal metabolism. To assure the safety of the patient's medication and inhibit the formation of thrombin, early venous anticoagulation is the prime therapy for patients with acute coronary syndromes. It antagonizes ongoing coronary thrombosis and promotes percutaneous coronary intervention to reduce mortality and acute stent thrombosis [7]. Although it can increase the blood clots, but not affect antithrombin of thrombin II inhibition of a factor. In addition, fondaparinux sodium does not interact with platelets, which does not affect the duration of bleeding. In recent years, a number of large trials abroad have proved that it is safe and effective for non-ST-segment elevation myocardial infarction with acute coronary syndrome [8] [9], which is superior to ordinary heparin or low molecular heparin significantly reducing bleeding and complications [10]. However, its efficacy and safety have not been proved for ST-segment elevation myocardial infarction patients [9]. In this paper, the research progress of fondaparinux sodium in acute coronary syndrome is reviewed and flow chart of literature screening as Figure 1.

\section{The Clinical Research of Fondaparinux on ST Segment Elevation Myocardial Infarction}

Tjeerd [11] researched the safety ang efficacy of fondaparinux compared to control according to 12,092 patients with ST-segment elevation myocardial infarction in the OASIS- 6 trial. OASIS- 6 is a controlled, randomized, double-blind trial, which is comparing fondaparinux to control (placebo or UFH).Myocardial infarction or death rates were reduced by fondaparinux at age 56 years ( $4.5 \%$ vs $4.8 \%$ ); at age 56 - 68 years ( $7.9 \%$ vs $9.7 \%$ ); at age $\geq 69$ years( $17.2 \%$ vs $19.8 \%)$. Severe hemorrhage rates were reduced at age 56 years $(0.5 \%$ vs $0.6 \%)$, at age 56 -

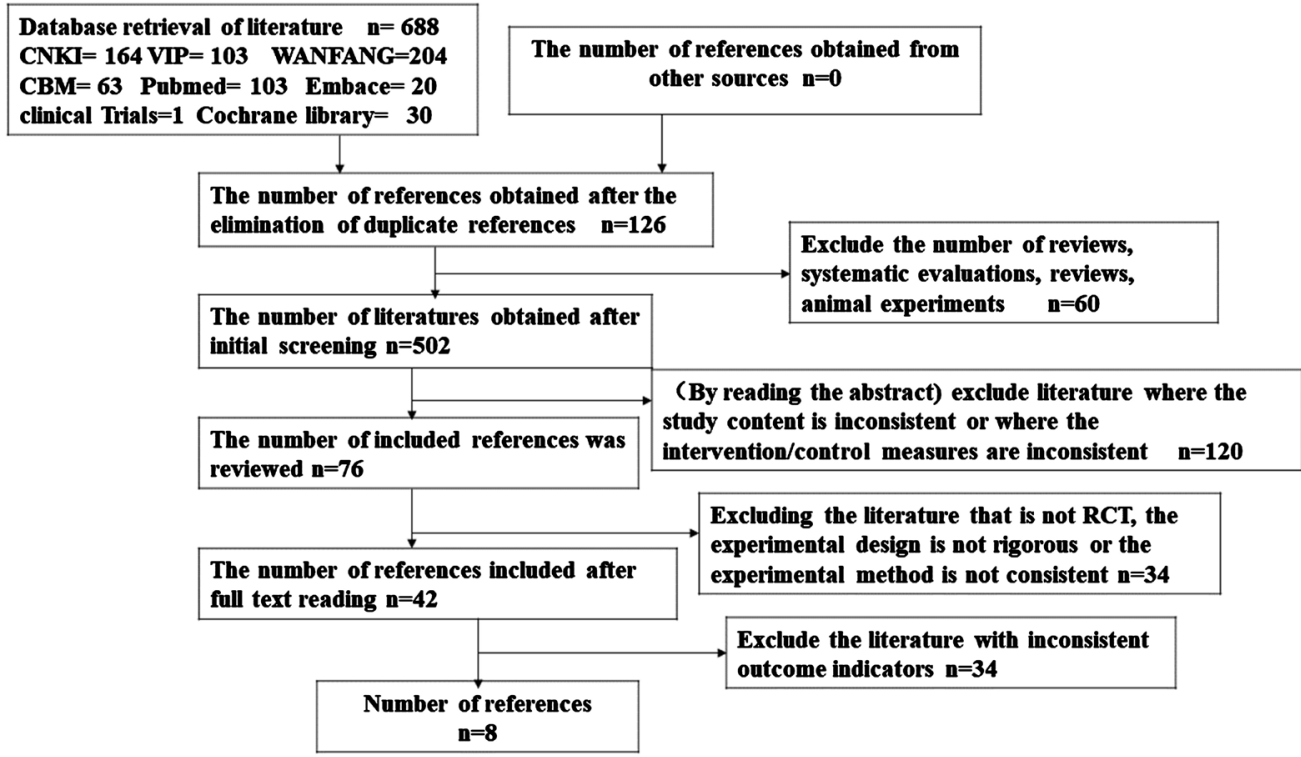

Figure 1. The flow chart of literature screening. 
68 years $(0.9 \%$ vs $1.5 \%)$, at age $\geq 69$ years $(2.1 \%$ vs $2.4 \%)$. Myocardial infarction, Death or severe bleeding rates were reduced at age 56 years ( $4.8 \%$ vs $5.0 \%)$, at age 56 - 68 years ( $8.1 \%$ vs $10.1 \%$ ), at age $\geq 69$ years ( $17.6 \%$ vs $20.4 \%)$. The benefits and risks of fondaparinux is coincident across every age level, supporting that fondaparinux's use across every age level of patients with ST segment elevation myocardial infarction who don't undergo primary percutaneous coronary intervention.

Alexandre de Matos Soeiro [12] study is a multicenter retrospective observational study. The study includes 2282 patients, which were divided into two groups (1947 in the enoxaparin group; 335 in the fondaparinux group). Firstly outcome was all-causes mortality. Secondary outcome was combined with reinfarction, cardiogenic shock, death, bleeding and stroke. Comparison was done through Chi-Square test and $\mathrm{T}$ test between the groups. With regards to treatment, the performance of a percutaneous coronary intervention in $35.1 \%$ in the enoxaparin group ( $\mathrm{p}=0.13$ ) and in $40.2 \%$ in the fondaparinux group. In the multivariate analysis, study respectively showed significant differences between two groups in relation to bleeding (2.3\% vs. $5.2 \%)$ and combined these events ( $13.8 \%$ vs. $22 \%)$. According to recently published international literature proved fondaparinux is superior to enoxaparin and can reduce the combined events and bleeding.

\section{The Clinical Research of Fondaparinux on NST Segment Elevation Myocardial Infarction}

Yusuf S [13] study 20,078 patients with acute coronary syndromes to receive either enoxaparin ( $1 \mathrm{mg} / \mathrm{kg}$ twice daily) or fondaparinux (2.5 $\mathrm{mg}$ daily) and assessed death, refractory ischemia, or myocardial infarction in nine days; major bleeding; and their combination. The period of Patient's following is six months. The primary-outcome events were similar in the enoxaparin and fondaparinux groups (579 with 573 with enoxaparin [5.7 percent] vs. fondaparinux [5.8 percent]). The events of meeting this combined outcome indicated a non-significant difference in the fondaparinux group at 30 days (805 vs. 864) and in the ending of the research (1222 vs. 1308). The rate of major bleeding in nine days was observably higher enoxaparin than with fondaparinux (412 events [4.1 percent] vs. 217 events [2.2 percent]). The all outcome and major bleeding at nine days supported fondaparinux (7.3 percent vs. 9.0 percent). Fondaparinux was showed a significantly reduction of deaths at 30 days (295 vs. 352 ) and at 180 days (574 vs. 638). Fondaparinux compared with enoxaparin in reducing the rate of ischemic events at nine days. However, fondaparinux can reduces major bleeding and improves long term morbidity and mortality.

Yan HB [14] studied 300 patients with NST segment elevation Acute Coronary Syndrome who were randomized to accepted either nadroparin ( $\mathrm{n}=150$, $0.1 \mathrm{ml} / 10 \mathrm{~kg} \mathrm{q} 12 \mathrm{~h})$ or fondaparinux $(\mathrm{n}=150,2.5 \mathrm{mg} / \mathrm{d})$ for a mean of 4 days, which is a randomized, prospective, single center, open-label study. The prime 
safety end-point was the events of major or minor bleeding in 9 days, which was not associated with coronary artery bypass grafting (CABG). The prime efficacy end-points contained myocardial infarction, death, or recurrent ischemia in 9 days. All patients were followed up 180-day. There was a non-significant $28 \%$ relative risk decrease in the prime safety end-point in fondaparinux group compared with group nadroparin (4.7\% vs. $6.7 \%)$. The prime efficacy end-point was $8.0 \%$ vs $10.0 \%$ in the group fondaparinux and nadroparin. The efficacy and safety end-points at 9 days ( $10.0 \%$ vs. $16.0 \%), 30$ days ( $14.0 \%$ vs. $17.9 \%)$, or 180 days $(18.7 \%$ vs. $27.3 \%)$ indicated a non-significant trend about a lower value in group fondaparinux. Fondaparinux showed a non-significant risk decrease in patients with NST segment elevation acute coronary syndrome in ischaemic both and bleeding events during short- and long-term follow-up comparing with nadroparin.

Coussement PK [15] study a total of 7 studies including 9618 patients (mainly non-ST-elevation myocardial infarction) were included. Analysis showed that the mortality of enoxaparin and fondaparinux sodium was similar (OR 1.05, 95\% CI 0.67-1.63, P = 0.84).There was no significant difference between myocardial infarction and stroke at different follow-up periods. However, the risk of total bleeding was significantly reduced in the fondaparinux heparin group (OR $0.47,95 \%$ CI $0.37-0.60, \mathrm{P}=0.00001$ ); during the 10-day follow-up, $\mathrm{P}=$ 0.00001). The risk of bleeding was still lower than that of enoxaparin at 30 days of follow-up or at the middle follow-up.

Sorosh [2] study showed low molecular weight heparin versus fondaparinux increase the risk of perioperative bleeding in patients who accepting coronary artery bypass. All acute coronary syndrome patients from the European multicenter registry, prospective on coronary artery bypass grafting preoperatively were treated with low-molecular weight heparin or fondaparinux. The prime outcome measure was major bleeding defined by the Universal Definition of Perioperative Bleeding stratified by $\mathrm{P} 2 \mathrm{Y}<$ sub $>12</$ sub $>$ inhibitor quit. Secondary outcome measures contained 3 other definitions of major bleeding, which was available in cardiac surgery trials. Bias score matching was performed to adjust for differences in preoperative and perioperative covariates, 1525 patients were included, of whom 1249 (81.9\%) low-molecular weight heparin and 276 (18.1\%) received fondaparinux preoperatively. In cohorts matched by propensity score (245 pairs), the risk of massive bleeding was similar between the fondaparinux and low-molecular weight heparin groups, which was based on the general definition of perioperative severe or massive bleeding (11.8\% vs $9.0 \%)$ and the other three major bleeding definitions. In summary, preoperative treatment with fondaparinux and low-molecular weight heparin was similar to perioperative bleeding in patients who with acute coronary syndromes undergoing coronary artery bypass grafting.

A recent study showed that non-ST patients were treated with fondaparinux and low molecular weight heparin in segmental elevation acute coronary syn- 
dromes in $15 \mathrm{~d}$ group recurrence of angina pain, $\mathrm{Q}$ wave myocardial infarction, emergency coronary intervention, death and bleeding cases. There was no significant difference between the molecular heparin group $(\mathrm{P}>0.05)$. This indicates that the therapeutic effect of fondaparinux on non - ST - segment elevation acute coronary syndrome is obvious [16].

However, PENTUA [17] study showed that the incidence of death, myocardial infarction or recurrent ischemia in subcutaneous injection of fondaparinux (and enoxaparin nine days) was $27.9 \%$ and $35.7 \%$. There was no difference in bleeding risk between the two groups $(\mathrm{P}<0.05)$. The Fondaparinux efficacy and safety of enoxaparin may be similar. The role of fondaparinux in acute coronary syndrome remains to be further studied [18].

Fondaparinux has been compared with low molecular weight heparins (LMWH) for Non-ST-Segment Acute Coronary Syndromes. We selected three random study information to systematically review the trials comparing the efficacy and safety of fondaparinux and LMWH for Non-ST-Segment Acute Coronary Syndromes (Table 1).

\section{Conclusion}

Acute coronary syndrome is characterized by acute onset, critical condition and high mortality, which need early anticoagulation intervention. The anticoagulant therapy is not only antithrombotic but also does not increase the risk of bleeding [19]. In terms of therapeutic drugs, the widely used antiplatelet drugs the anticoagulant and aspirin have gradually become routine treatment drugs [20]. The combination of antiplatelet agents and anticoagulants observably decreases the events of severe cardiovascular diseases and mortality. But the side-effect of anticoagulant drugs is also caused by an observably increase in various bleeding [7]. The consequences of serious threating to the patient's life and health are increasing at the same time. A large number of clinical studies have been conducted on fondaparinux by researchers, which showed that fondaparinux can decrease the risk of mortality, bleeding and morbidity for non-ST-segment elevation acute coronary syndrome. Fondaparinux may be the better choice, and this result is mainly applicable to patients with non-ST-segment elevation acute coronary syndrome [21]. But fondaparinux is III class international guidelines

Table 1. Three large random studies on non-ST-segment elevation acute coronary syndromes.

\begin{tabular}{cccccccccc}
\hline \multicolumn{3}{c}{ Characteristics of Included Studies } & \multicolumn{2}{c}{ Medication Time } & \multicolumn{2}{c}{ Doses of Medication } & Number of Patients \\
\hline Study & Year & $\mathbf{N}$ & Clinical Trail Setting & Fondaparinux & LMWH & Fondaparinux & LMWH & Fondaparinux & LMWH \\
\hline OASIS-5 & 2006 & 20078 & $\begin{array}{c}\text { Multicentered, double } \\
\text { blind RCT }\end{array}$ & 8 Day & $2-8$ Day & $2.5 \mathrm{mg} \mathrm{qd}$ & $1 \mathrm{mg} / \mathrm{kg} \mathrm{bid}$ & 10021 & 10057 \\
PENTUA & 2004 & 459 & $\begin{array}{c}\text { Multicentered, double } \\
\text { blind RCT }\end{array}$ & $3-7$ Day & $3-7$ Day & $2.5 \mathrm{mg} \mathrm{qd}$ & $1 \mathrm{mg} / \mathrm{kg} \mathrm{bid}$ & 229 & 230 \\
Yan & 2011 & 300 & $\begin{array}{c}\text { prospective, open-label, } \\
\text { and single center study, } \\
\text { RCT }\end{array}$ & 4 Day & 4 Day & $2.5 \mathrm{mg} \mathrm{qd}$ & $0.01 \mathrm{ml} / \mathrm{kg} \mathrm{q12}$ & 150 & 150 \\
\hline
\end{tabular}


that recommend not advocating using for ST-segment elevation acute coronary syndrome patients. However, the limitation number of patients with analysis that needs further large randomized trials will be confirmed. It is difficult to completely avoid clinical therapy. The influence of subjective choice on the results of the study was further evaluated. Large randomized controlled studies are needed to study the safety and efficacy of fondaparinux.

\section{Conflicts of Interest}

The authors declare no conflicts of interest regarding the publication of this paper.

\section{References}

[1] Siddiqui, M.U., Chiuzan, C., Siddiqui, M.D., Ali, S.S., Naeem, Z. and Islam, S. (2020) Temporal Pattern of CABG and PCI after Non-ST Elevation Myocardial Infarction among Elderly Patients from NHDS. Cureus, 12, e6814.

[2] Sorosh, K., et al. (2019) Perioperative Bleeding in Patients with Acute Coronary Syndrome Treated with Fondaparinux versus Low-Molecular-Weight Heparin before Coronary Artery Bypass Grafting. The American Journal of Cardiology, 123, 565-570. https://doi.org/10.1016/j.amjcard.2018.11.028

[3] Zeymer, U., Rao, S.V. and Montalescot, G. (2016) Anticoagulation in Coronary Intervention. European Heart Journal, 37, 3376-3385. https://doi.org/10.1093/eurheartj/ehw061

[4] Kyle, F., Pharma, D.P., Wang, D. and Sargent, K. (2019) Successful Use of Fondaparinux in the Setting of Heparin-Induced Thrombocytopenia with Thrombosis Confirmed by Serotonin-Release Assay and Factor V Leiden. Journal of Clinical Pharmacy and Therapeutics, 44, 809-812. https://doi.org/10.1111/jcpt.12994

[5] Zeitouni, M., Kerneis, M., Nafeeb,T., Colleta, J.-P., Silvaina, J. and Montalescot, G. (2018) Anticoagulation in Acute Coronary Syndrome-State of the Art. Progress in Cardiovascular Diseases, 60, 508-513.https://doi.org/10.1016/j.pcad.2018.01.004

[6] Shah, D., Risebrough, N.A., Perdrizet, J., Iyer, N.N., Gamble, C. and Dang-Tan, T. (2018) Cost-Effectiveness and Budget Impact of Liraglutide in Type 2 Diabetes Patients with Elevated Cardiovascular Risk: A US-Managed Care Perspective. Clinicoeconomics and Outcomes Research, 10, 791-803.

https://doi.org/10.2147/CEOR.S180067

[7] Galli, M., Andreotti, F., D’Amario, D., Vergallo, R., Vescovo, G.M., Giraldi, L., Migliaro, S., Ameri, P., Porto, I. and Crea, F. (2020) Antithrombotic Therapy in the Early Phase of Non-ST-Elevation Acute Coronary Syndromes: A Systematic Review and Meta-Analysis. European Heart Journal-Cardiovascular Pharmacotherapy, 6, 43-56. https://doi.org/10.1093/ehjcvp/pvz031

[8] Geisler, T., et al. (2016) Impact of Anticoagulation Regimen Prior to Revascularization in Patients with Non-ST-Segment Elevation Acute Coronary Syndromes: The Acuity Trial. Catheterization \& Cardiovascular Interventions, 88, 174-181. https://doi.org/10.1002/ccd.26232

[9] Wang, J., et al. (2005) Evaluation of the Safety and Efficacy of High-Dose Tirofiban in Direct Percutaneous Coronary Intervention in Patients with Acute ST-Segment Elevation Myocardial Infarction. Chinese General Practice, No. 5, 3276-3283.

[10] NCT (2017) Safety and Efficacy of LMWH versus Rivaroxaban in Chinese Patients 
Hospitalized with Acute Coronary Syndrome.

[11] Van Rees Vellinga, T.E., et al. (2010) Efficacy and Safety of Fondaparinux in Patients with ST-Segment Elevation Myocardial Infarction across the Age Spectrum. Results from the Organization for the Assessment of Strategies for Ischemic Syndromes 6? (OASIS-6) Trial. American Heart Journal, 160, 1049-1055. https://doi.org/10.1016/j.ahj.2010.08.038

[12] De Matos Soeiro, S., et al. (2016) Fondaparinux versus Enoxaparin-Which Is the Best Anticoagulant for Acute Coronary Syndrome?-Brazilian Registry Data. Arquivos Brasileiros de Cardiologia, 107, 239-244.

[13] The Fifth Organization to Assess Strategies in Acute Ischemic Syndromes Investigators (2006) Comparison of Fondaparinux and Enoxaparin in Acute Coronary Syndromes. The New England Journal of Medicine, 354, 1464-1476. https://doi.org/10.1056/NEJMoa055443

[14] Yan, H.-B., et al. (2011) Comparison of Safety and Efficacy between Fondaparinux and Nadroparin in Non-ST Elevation Acute Coronary Syndromes. Chinese Medical Journal, 124, 879-886.

[15] Coussement, P.K., Bassand, J.-P., Convens, C., Vrolix, M., Boland, J., Grollier, G., Michels, R., Vahanian, A., Vanderheyden, M., Rupprecht, H.-J. and Van de Werf, F. for the FENTALYSE Investigators (2001) A Synthetic Factor-Xa Inhibitor (ORG31540/SR9017A) as an Adjunct to Fibrinolysis in Acute Myocardial Infarction. The Pentalyse Study. European Heart Journal, 22, 1716-1724. https://doi.org/10.1053/euhj.2001.2777

[16] Yiran, Z., Zhang, M., Tan, L., Pan, N. and Zhang, L. (2019) The Clinical Use of Fondaparinux: A Synthetic Heparin Pentasaccharide. Progress in Molecular Biology and Translational Science, 163, 41-53.

[17] Simons, M.L., et al. (2004) A Dose-Finding Study of Fondaparinux in Patients with Non-ST-Segment Elevation Acute Coronary Syndromes: The Pentasaccharide in Unstable Angina (PENTUA) Study. Journal of the American College of Cardiology, 43, 2183-2190. https://doi.org/10.1016/j.jacc.2004.02.051

[18] Li, S., et al. (2015) Comparison of Safety and Efficacy of Sodium Sulfonate and Nachorheparin in Patients with Non-ST-Segment Elevation Acute Coronary Syndrome during Perioperative Interventional Therapy. China Circulation Journal, 30, 111-115.

[19] Qiao, J., Zhang, X., Zhang, J., Li, P., Xu, B., Wang, S., Jiang, H., Shen, Y. and Wang, K. (2016) Comparison between Fondaparinux and Low-Molecular-Weight Heparin in Patients with Acute Coronary Syndrome: A Meta-Analysis. Cardiology, 133, 163-172. https://doi.org/10.1159/000441442

[20] Clemens, A., Fraessdorf, M. and Friedman, J. (2013) Cardiovascular Outcomes during Treatment with Dabigatran: Comprehensive Analysis of Individual Subject Data by Treatment. Vascular Health and Risk Management, 9, 599-615. https://doi.org/10.2147/VHRM.S49830

[21] Tong, J.P. (2020) Application of Calcium Nachor Heparin and Sodium Sulfonda in the Prevention of Postoperative Deep Vein Thrombosis in Patients with Spinal Fractures. Contemporary Chinese Medicine, No. 5, 88-91. 